\title{
Transient groundwater flow in a single fracture
}

\author{
M. Polák \& J. Mls \\ Institute of Hydrogeology, Engineering Geology and Applied Geophysics, \\ Charles University, Czech Republic
}

\begin{abstract}
Understanding the water flow behaviour in a single fracture is essential for investigating groundwater flow and solute transport in fractured crystalline rock formations in the context of water supply, remediation of dissolved contaminant or projecting long-term nuclear waste repositories. Numerical modelling was used as a tool for studying the geometrical and hydraulic properties of a single fracture. Two computer codes were developed for this purpose. The first one simulates the fracture-free space geometry and the other one solves the transient flow equation. The fracture geometry simulation consists of defining the contact zones where the fracture is closed for the water flow, and of generating the aperture values in zones of water flow. The distribution of contact areas within the simulated fracture is governed by principles of percolation theory. The positive values of aperture in water-flow zones were distributed according to the knowledge of real fracture data. Transient flow simulation is based on principles of fluid mass balance in the fracture space and on locally valid cubic law that quantifies flow rate through the fracture profile. The problem is solved numerically by means of the discretization in time and the finite element method. The percolation theory approach makes it possible to get a faithful evaluation of the fracture permeability and the transient form of the flow equation enables us to simulate the flow and pressure field propagation in accordance to laboratory or field test conditions.

Keywords: modelling, fracture geometry, transient flow, hydraulic head.
\end{abstract}

\section{Introduction}

Research of the groundwater flow through fractured rock has increased in the last three decades. A lot of mathematical modelling work has been concerned with characterization of such natural environment. Basic approaches to the water flow 
in the fractured media can be divided into two main groups. For the modeling of the medium containing a lot of interconnected fractures, the equivalent continuum approach is suitable. Effective use of this conceptual model supposes extremely dense fracture network that causes homogenization of environment. Otherwise a discrete fracture model will be more appropriate. Analyzing of fluid flow behaviour in a single fracture is essential for understanding of flow mechanism in the fracture networks. There are three modeling approaches to describe single fracture geometry. The parallel plate model is simple but it is not able to describe spatial heterogeneity of inner fracture space [1]. The flow tube model is characterized by incorporating of circular or rectangular tubes within the fracture plane. It was shown that the real flow channels within the fracture are not fixed but they change with the direction of potential gradient [2]. The variable aperture approach includes aperture variation in whole fracture plane. This conceptual model is the best fit of real fracture conditions. Aperture is usually generated by variety of stochastic random functions. Usually the fracture void space geometry is represented by random three-dimensional functions which describe the topology of surfaces that confine the free fracture space and determine its flow and transport properties [3]. In the presented work we have used this model approach. In the single fracture consisting of two parallel surfaces the flow is governed by "cubic law" (Hagen-Poiseuille's equation). The important implication finding is that fluid flow through the fracture may be fully characterized by aperture, although the velocity varies across that distance [4]. Using the cubic law for solution of the fluid flow in more natural fracture with variable aperture is based on assumption of local validity of this equation in each segment of the fracture. We used this principle to investigate the flow in a single fracture. Some authors [5-7] found that using locally held cubic law overestimates the flow rate through fracture in the range from 1.3 times to 1.9 times.

\section{Problem definition}

Mathematical modeling tools were used for analyze the pressure changes inside the void fracture space in a laboratory rock sample (figure 1) before and after the steady state flow was established. We used mathematical model of transient flow for the estimation of time that is needful for setting the pressure equilibrium in the fracture up. Tested sample of granite has shape of a cube with the size of edge about $0.6 \mathrm{~m}$. There is one visible fracture with a slope of about $7^{0}$ that divides the sample almost in the middle into two parts. Fracture was sealed on the surface of the sample except of two gabs that were used for steady state flow field creation. One borehole drilled into the sample was used for water injection during the test.

\section{Fracture simulation}

A computer code was developed for the single fracture space simulation. The fracture geometry simulation consists of two main parts. The first part defines 
the contact zones where the walls of the fracture are in direct contact to each other and make these fracture areas closed for the water flow. The second part generates the fracture aperture in zones accessible for flowing water.

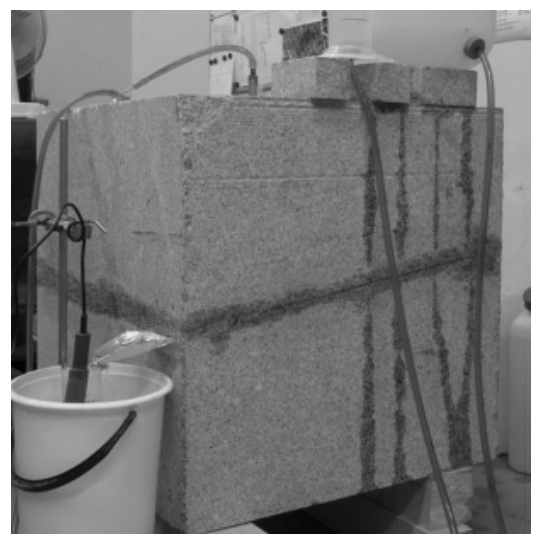

Figure 1: Laboratory sample of the fractured rock.

\subsection{Fracture plane generation}

Fracture permeability is sensitive to applied normal stress. Especially in the deep parts of crystalline massifs, the normal stress causes the closure of significant parts of fractures. The distribution of contact areas within the simulated fracture in our model is governed by principles of percolation theory and random numbers generation. Some authors, i.e. Mourzenko et al. [3] or Renshaw [8], have suggested that the real fractures have fractal properties. Veselý and Mls [9] studied minimal requirements on the element number in percolation meshes in order to obtain statistically relevant percolation cluster formations.

Two-dimensional plane fracture is simulated by rectangular tetragon. This tetragon represents central plane of the fracture. It is discretised into finite number of the linear triangular elements. The program makes use of the percolation theory and defines the inner fracture structure based on percolation probability $p$. Each triangle of the mesh is open with probability $p$ and closed with probability 1-p. Any group of adjacent open triangles that is large enough for connection of the opposite sites of the system forms a percolation cluster. Closed triangles are labeled as primarily closed. If percolation cluster appears others triangles are closed due to blocking of pathways by primarily closed triangles. These triangles are labeled as secondarily closed. All nodes included in closed triangles are labeled as closed. If two adjacent nodes are closed the bond between them is labeled as closed too and it is impermeable for flowing water. Fracture plane generated according to probability $\mathrm{p}=0.92$ is shown in figure 2 .

\subsection{Aperture generation and fracture orientation}

The positive values of aperture in water flow zones of the simulated fracture were distributed according to the knowledge of real fracture data $[5,10]$ and 
according to the apertures range that was calibrated by a steady state model of hydraulic laboratory test. All nodes labeled as closed got zero apertures. Open nodes have randomly assigned aperture value according to the exponential probability distribution in the range 40-1200 $\mu \mathrm{m}$ (figure 3). Fracture aperture is distributed symmetrically along the central fracture plane.

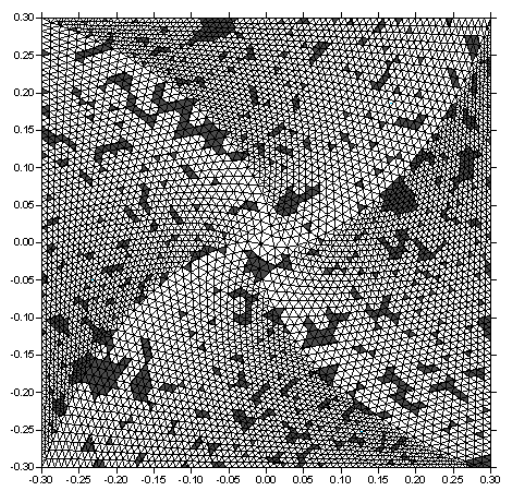

Figure 2: Central fracture plane discretised into 8743 active triangular elements.

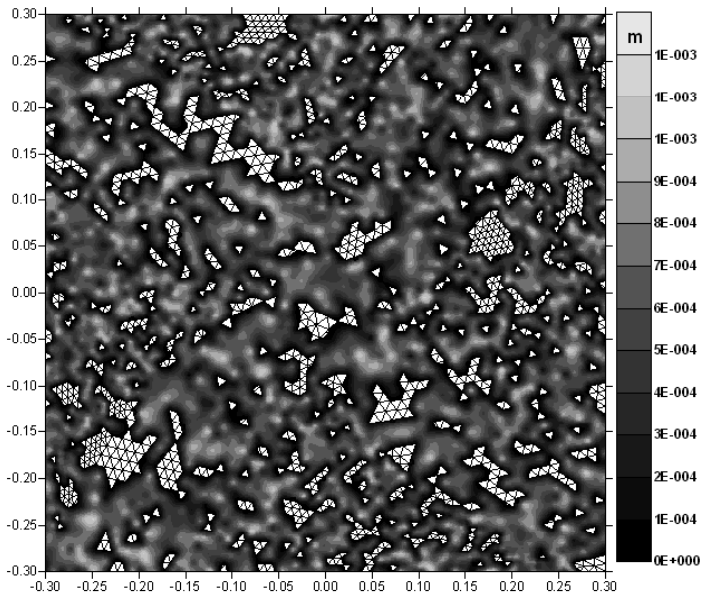

Figure 3: Random aperture distribution within the generated fracture.

The last step in fracture generation process is to set orientation of the fracture. The simulated fracture can be arbitrarily rotated around the horizontal axes. In our case the rotation was $7^{0}$ around the $y$ axis. 


\section{Transient flow simulation}

\subsection{Theory}

Transient flow simulation in randomly generated fracture is based on two basic principles. Principle of mass conservation of the fluid in the void fracture space, eqn. (1), and principle of locally valid cubic law that quantifies flow rate perpendicular to the fracture profile eqn. (2). Principle of mass conservation is valid in each point in the fracture and can be written as follows:

$$
\sigma d(x) \frac{\partial p}{\partial t}(x, t)+\operatorname{div} \quad q(x, t)=0,
$$

where $x=\left(x_{1}, x_{2}\right)$ are coordinates in the fracture plane, $p$ is the pressure inside the fluid, $d$ is aperture perpendicular to the central fracture plane, $\sigma$ is specific storage of the fracture and $q=\left(q_{1}, q_{2}\right)$ is specific flow rate across transversal line perpendicular to the central fracture plane. Hagen-Poiseuille's equation (cubic law) is expressed as follows:

$$
q_{i}(x, t)=-\frac{d^{3}(x)}{12 \mu}\left(\rho(x, t) g \frac{\partial z}{\partial x_{i}}+\frac{\partial p(x, t)}{\partial x_{i}}\right), i=1,2,
$$

where $z$ is vertically upward oriented coordinate, $g$ is gravitational acceleration, $\rho$ is density and $\mu$ is dynamical viscosity of the fluid in the fracture. We assumed constant value of fluid density $\rho$. Then the eqn. (3) can be written as follows:

$$
q(x, t)=-\frac{\rho g d^{3}(x)}{12 \mu} \operatorname{div} \quad u(x, t),
$$

where $u(x, t)$ is hydraulic head expressed:

$$
u=z+\frac{p}{\rho g} .
$$

As a result of substitution of eqn. (3) into the eqn. (1) the governing equation for fluid flow in the fracture is set up and it can be written as follows if the summative rule is used:

$$
\sigma d(x) \frac{\partial p}{\partial t}(x, t)=\frac{\partial}{\partial x_{i}}\left(\frac{\rho g d^{3}(x)}{12 \mu} \frac{\partial u}{\partial x_{i}}\right) .
$$

In the randomly generated fracture the governing equation (5) is solved together with boundary condition of $1^{\text {st }}$ and $2^{\text {nd }}$ type, eqn. (6), by finite element method.

$$
u(x, t)=\text { const., } \frac{\partial u(x, t)}{\partial x}=0 .
$$

Rothe's method has been used to solve initial boundary value problems with this equation.

\subsection{Boundary conditions and storativity}

There were two parts of the fracture outer boundary where stable boundary condition of constant hydraulic head was established. These two parts (in upper left corner and lower right corner) represent the gaps used for inflow and outflow 
of the water. Constant value of hydraulic head was prescribed according to the elevation of these boundaries above the reference plane. The rest of outer boundary was assigned as no-flow. During the first $10 \mathrm{~s}$ of the simulation (for time $t>0 \mathrm{~s}$ ), that represent water injection, the constant head of $0.3 \mathrm{~m}$ was assigned into the nodes of the mesh which represent intersection of the fracture and injection borehole. For simulation time $t>60 \mathrm{~s}$, this boundary condition was removed.

An important parameter of any simulation is the storativity. In the general case, the water is assumed to be compressible and the solid matrix is deformable and, hence, the changes in pressure will cause the water content to vary with time. The value of the specific storativity of the fracture space used in our simulation was $1 \times 10^{-3} \mathrm{~Pa}^{-1}$.

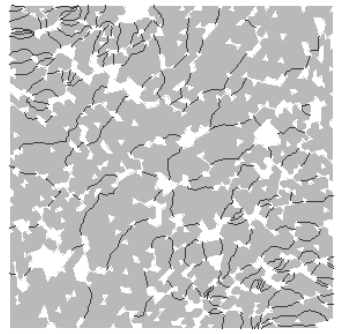

simulation time $t=0 \mathrm{~s}$

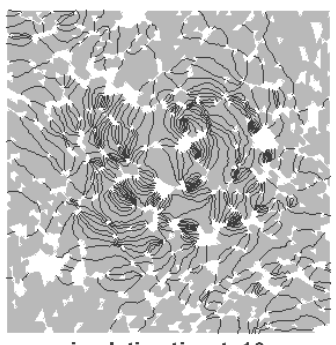

simulation time $t=12 \mathrm{~s}$

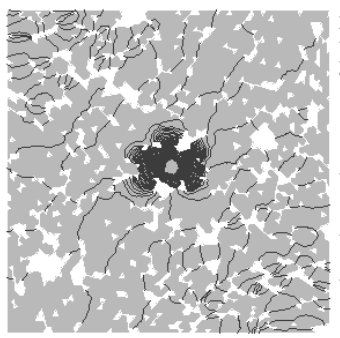

simulation time $t=0.01 \mathrm{~s}$

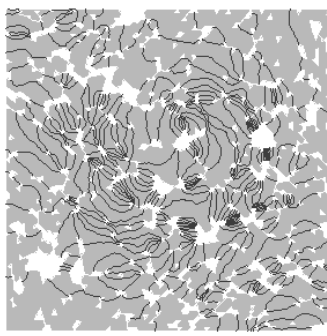

simulation time $t=1 \mathrm{~h}$

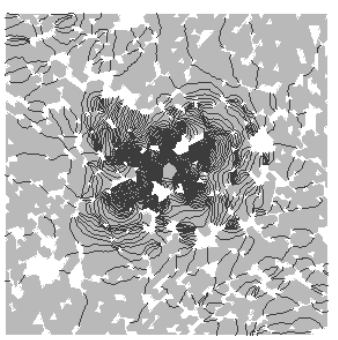

simulation time $t=10 \mathrm{~s}$

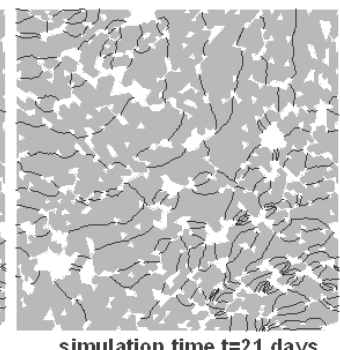

Figure 4: Hydraulic head in specific simulation times.

\subsection{Simulation results}

From analysis of the hydraulic head distribution in different times of the simulation, it is possible to determine the time that is necessary for pressure field stabilization. During the injection stage of simulation, the time necessary to reach the steady-state flow was 8 seconds. After that, the changes in the distribution of hydraulic head were negligible. The process of the pressure field rebalancing is much longer. Simulations show that the second stage, the rebalancing, takes almost 22 days. Absolute balancing of the hydraulic head to the original state takes more than 100 days. Changes in the hydraulic head field for specific simulation times are documented in figure 4 . The estimation of 
velocity distribution in the void fracture space is shown in figure 5 . In the simulation time $\mathrm{t}=0 \mathrm{~s}$ the velocity in the fracture ranged between $0 \mathrm{~m} / \mathrm{s}$ and $0.15 \mathrm{~m} / \mathrm{s}$ (in the triangles with nodes with inflow boundary condition). The highest velocity during simulation was calculated at the very beginning of the injection stage of simulation. Its estimation was over $3 \mathrm{~m} / \mathrm{s}$ in some triangles with bigger aperture close to the injection borehole. During the simulation time the velocity decreased and the zones of heightened velocity level moved through the main pathways.

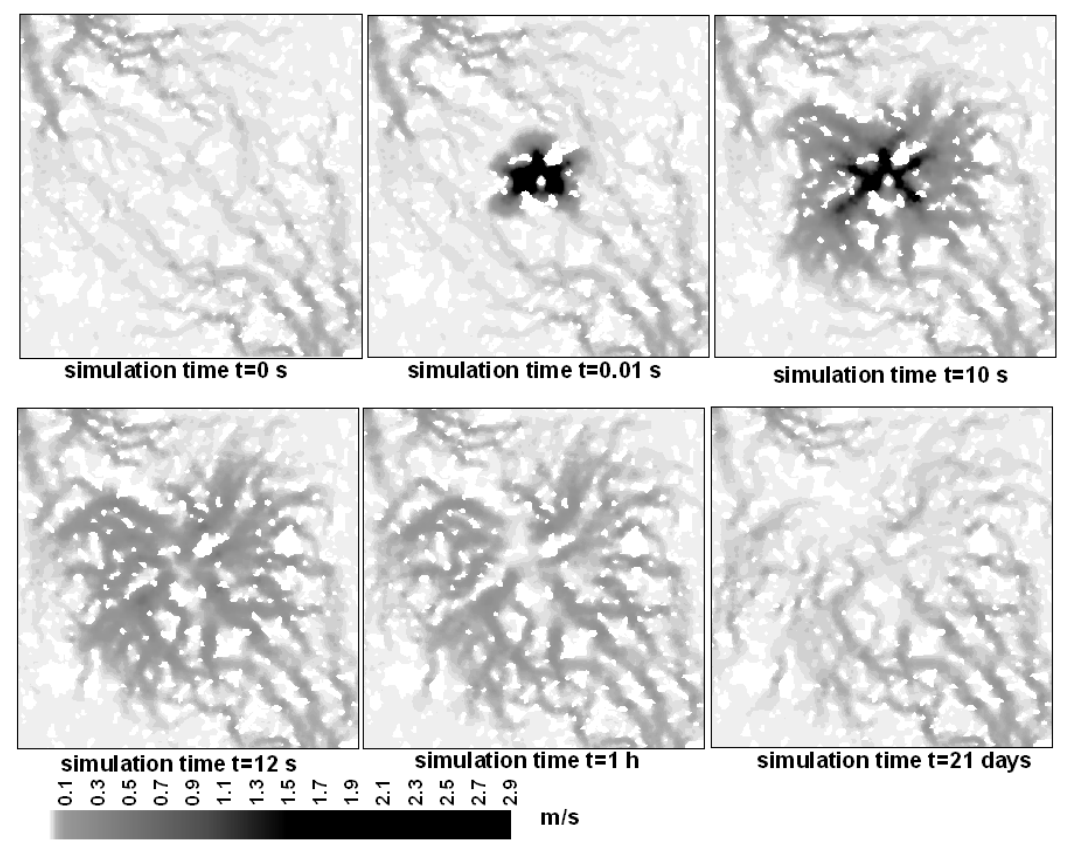

Figure 5: Velocity distribution in specific simulation time.

\section{Conclusions}

Two numerical models were developed and used to perform simulation of a single fracture geometry and transient flow calculation. These codes were used for estimation of time dependent distribution of the hydraulic head before and after the steady-state flow was established in the laboratory tests. Simulation results show that changes in pressure are balanced very quickly after injection stage of test has started. Much longer time is necessary for the new balance after removing the injection. More than 20 days are necessary for fracture pressure rebalancing. Adjusted results show that the described method can be simply and effectively employed for fracture flow investigation. 


\section{Acknowledgements}

The presented work was supported by Czech science foundation under grant No. 205/04/0614 and by the Ministry of Industry and Trade under project No. $1 \mathrm{H}$ PK/31 MPO ČR.

\section{References}

[1] Abelin, H. \& Birgersson, L. \& Gidlund, J. \& Neretnieks, I., A Large-Scale Flow and Tracer Experiment in Granite. 1. Experimental Design and Flow Distribution. Water Resour. Res., 27(12), pp. 3107-3117, 1991.

[2] Tsang Y.E. \& Tsang C.F., Flow channeling in a single fracture as a two dimensional strongly heterogeneous permeable medium, Water Resour. Res., 33, pp. 2076-2080, 1989.

[3] Mourzenko, V.V.\& Thovert, J.-F. \& Adler, P. M., Geometry of simulated fractures, Phys. Rev. E, 53(6), pp. 5606-5625, 1996.

[4] Ge, S., A governing equatition for fluid flow in rough fractures, Water Resour. Res., 33(1), pp. 53-61, 1997.

[5] Hamaki, E. \& Larsson, E., Aperture measurements and flow experiments on a single natural fracture, Int. J. Rock Mech. Min. Sci. Geomech Abstr., 33(4), pp. 395-404, 1996.

[6] Nichol N. J. \& Rajaram H. \& Glass R.J. \& Detwiler R., Saturated flow in a single fracture: Evaluation of the Reynolds equation in measured aperture fields, Water.Resour.Res., 35(11), pp. 3361-3373, 1999.

[7] Konzuk, J. S. \& Kueper, B.H., Evaluation of cubic law based models describing singe-phase flow through a rough-walled fracture, Water Resour. Res., 40, W02402, 2004.

[8] Renshaw, C. E., Influence of subcritical fracture growth on the connectivity of fracture works. Water Resour. Res., 32(6), pp. 1519-1530, 1996.

[9] Veselý, M., Mls, J., Water Flow in a Single Fracture with Variable Aperture, Journal of Hydrology and Hydromechanics, 2, pp. 77-90, 2004.

[10] Pyrak-Nolte \& L.J. \& Myer, L.R. \& Cook, N.G.W. \& Witherspoon, P.A., Hydraulic and Mechanical Properties of Natural Fractures in Low Permeability Rock, Proc. of the Sixth Int. Congress on Rock Mechanics, eds. G. Herget and S. Vongpaisal, Montreal, Canada, pp. 225-231, 1987. 